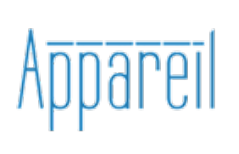

Appareil

$5 \mid 2010$

Identités de la guitare électrique

\title{
Ce qui se passe dans tes doigts
}

Entretien avec le guitariste électrique Claude Pavy

\section{Santiago Quintans et Otso Lähdeoja}

\section{(2) OpenEdition}

\section{Journals}

Édition électronique

URL : http://journals.openedition.org/appareil/1012

DOI : 10.4000/appareil. 1012

ISSN : 2101-0714

Éditeur

MSH Paris Nord

Référence électronique

Santiago Quintans et Otso Lähdeoja, « Ce qui se passe dans tes doigts », Appareil [En ligne], 5 | 2010, mis en ligne le 12 avril 2010, consulté le 30 juillet 2020. URL : http://journals.openedition.org/appareil/ 1012 ; DOI : https://doi.org/10.4000/appareil.1012

Ce document a été généré automatiquement le 30 juillet 2020.

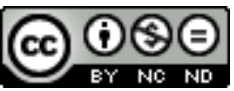

Appareil est mis à disposition selon les termes de la Licence Creative Commons Attribution - Pas d'Utilisation Commerciale - Pas de Modification 4.0 International. 


\title{
Ce qui se passe dans tes doigts
}

\author{
Entretien avec le guitariste électrique Claude Pavy
}

\author{
Santiago Quintans et Otso Lähdeoja
}

Une question simple, c'est quoi la guitare électrique?

Claude Pavy : C'est une planche avec un manche, des cordes qui font tzing, des micros qui captent tzing, et après on fait joujou avec ça.

La guitare électrique c'est d'abord le son. Je dis souvent à mes élèves : « la guitare, cela ne m'intéresse pas ». Alors évidemment, ils me regardent avec des yeux ronds, et ils me disent, "T'es guitariste?» Oui, mais la guitare en elle-même, cela ne m'intéresse pas. Ce qui m'intéresse, c'est ce qu'on peut faire avec, c'est-à-dire de la musique. C'est la musique, le son de la guitare, mais pas la guitare elle-même... j'écoute d'ailleurs très peu de guitaristes. En général, ils m'ennuient. Les doigts qui bougent et tout ça, cela ne m'a jamais passionné. C'est mon instrument, donc, il faut travailler, il y a de la technique à maîtriser. Ce qui m'intéresse ce sont les caractéristiques sonores pour faire de la musique, mais pour moi la guitare électrique, c'est un outil, c'est un instrument, pas autre chose.

La guitare électrique a donc une particularité sonore?

Claude Pavy: Oui bien sûr, par exemple la guitare classique ne me touche pas beaucoup, et notamment la littérature pour guitare classique, car, malheureusement, peu de compositeurs se sont intéressés à l'instrument. Je ne suis pas le seul à dire cela. Évidemment il y a Brouwer, les Sud-Américains, Villa-Lobos, plus moderne, plus populaire, plus vivant... Mais ce n'est pas de cela dont on va parler.

Concernant la guitare électrique, tu évoques souvent la pédale de volume comme faisant partie de l'instrument.

Claude Pavy : Complètement, la guitare électrique, c'est le son particulier qu'on peut tirer de cet objet. Ce qui m'a donné envie de jouer de cette guitare, c'est en 1959-1960, Apache des Shadows, quand ce son-là est arrivé, une guitare claire, avec de l'espace et de la réverb, c'était nouveau, on n'avait jamais entendu ça avant. Jusque-là, la guitare, c'était blinb bling bling à la Brassens, un peu de guitare jazz ronron, Wes Montgomery et tout ça, avec un son toujours le même, bien rond mais sans beaucoup de 
personnalité (je ne parle pas de la qualité du jazzman qui est extraordinaire), mais le son en lui-même, je ne l'ai jamais trouvé passionnant. La guitare électrique, c'était plutôt une guitare amplifiée, avec un son un peu saxophone, un peu ronron, mais au niveau de la sonorité, je n'ai jamais trouvé cela captivant. Par contre quand sont arrivés avec les Shadows, guitares claires, réverb, espace, brillance, profondeur, des sons variés, j'ai fait « waouw ! » Au départ, c'est ça qui m'a donné envie de faire de la guitare électrique, c'est le son!

Oui, le son est produit par plusieurs choses...

Claude Pavy : La première petite guitare que j'avais, je me disais, mais comment font les Shadows pour avoir ces sons-là? Il y avait pas que de la réverb, il y avait un petit écho. Finalement on a compris.

Après j'ai fait un petit peu d'études d'électronique, cela m'a aidé, mais j'étais dans une très mauvaise école, donc je me suis orienté vers la guitare électrique. Connaître l'électronique m'a finalement aidé pour toute la partie électrique de la guitare, les bases du courant alternatif, souder des câbles; depuis 40 ans, c'est moi qui fais tout mon matériel...

Est-ce que tu penses que cette compétence technique t'a permis d'être plus précis dans le son?

Claude Pavy : Oui, avoir certaines notions de base, savoir ce qu'est une fréquence, un spectre, une phase, c'est très utile. Connaître les principes physiques de base, comment marche un micro, un transformateur, un signal, un condensateur, un bobinage, les principes élémentaires de base du traitement du son, la physique de base, oui cela m'a servi, surtout en studio, je pouvais facilement parler avec les techniciens. Toutes les années soixante, soixante-dix quatre-vingt, j'étais alors un requin de studio ; les musiciens, même les guitaristes, n'avaient aucune compétence en la matière. Beaucoup de guitaristes professionnels, même de nos jours, ne savent pas comment vibre une corde, comment est conçue une guitare, comment elle doit être accordée... ils ont souvent une mauvaise réputation dans le métier, car c'est un instrument qui est souvent faux. J'étais comme ça au début (et d'ailleurs à l'époque, c'était la mode des 12 cordes!); et puis un jour, je me suis servi de mes petites connaissances techniques. Une fois que j'ai compris qu'il fallait s'accorder au tempérament égal et non pas naturel... voilà un exemple... Et c'est comme ça que je me suis fait une réputation professionnelle en studio.

La guitare électrique est un instrument qui permet d'avoir des sons clairs, sombres, pincés, moelleux, agressifs, doux, rêveurs... On a une palette sonore qui est formidable pour un compositeur, pour un orchestrateur. Cette richesse sonore permet de créer des univers, des ambiances, avec, ce que n'a pas un synthé, malgré tout, cette chose irremplaçable qui est la production physique et naturelle du son sur la corde de la guitare. Il ne faut pas oublier les doigts, l'instrument, le toucher, cette chaleur et cette sensibilité-là qu'on va traiter après. Pour moi c'est primordial. C'est pour cela que je n'aime pas le synthé-guitare, où le manche devient une sorte de clavier pour synthé. Où la personnalité de celui qui joue est gommée. Il existe des claviers sensitifs, mais c'est très fruste, du fait de midi. La courbe midi à 127 pas, c'est faible pour vraiment travailler sur la dynamique; dans les premiers pas de l'échelle, on entend vraiment les marches d'escalier. C'est d'ailleurs aussi un problème qu'on trouve sur les pédales de volume du marché, mais posé d'une autre façon : les courbes sont conçues pour répondre au besoin du marché, qui vise le grand public des 
guitaristes électriques. Les gens comme nous, qui cherchent la finesse, l'expressivité musicale, ne représentent que $0,5 \%$ du marché, c'est-à-dire rien.

La guitare électrique a son propre toucher, sa propre sonorité, qui a à voir avec la guitare classique ce que le clavecin a à voir avec l'orgue. Ce sont deux instruments différents, le matériau sonore n'est pas le même. Le nylon et l'acier ne réagissent pas pareil, l'enveloppe du son, le sustain, la richesse harmonique, sont autres. Comment vit le son, la corde, la résonance, c'est très différent.

Par rapport à ces histoires de techniques, la guitare électrique vient en grande partie du domaine rock et du jazz amplifié. Pour moi la guitare électrique s'impose avec la Stratocaster, une invention formidable dans la forme, équilibrée et dans les idées qu'il y a dedans.

Quand tu t'es approprié cet instrument, pour jouer une autre musique, quelles adaptations techniques de jeu as-tu faites, l'as-tu personnalisé ?

Claude Pavy : Pas du tout, que je joue un bon rock, un blues ou une pièce de Hugues Dufourt comme la Cité des Saules, pour moi il n'y a pas de différence. À chaque fois, je vais essayer de trouver la sonorité, donc la manière d'attaquer les réglages sur l'instrument, comment faire vivre le son pour aller dans l'esprit du discours musical. Croire que les solutions pour résoudre des problèmes sont dans la maîtrise de la technique, c'est très facile, en lisant les journaux sur la guitare. Mais c'est loin de la réalité musicale.

Ce que j'aimerais souligner, c'est la dimension orchestrale de cet instrument.

Ce qui est intéressant, c'est le son. La guitare électrique, c'est une planche. Avec un manche le mieux possible, avec un peu de lutherie, des micros de différents types, et un vibrato.

Le son, grâce aux micros, au traitement, c'est cela que j'appelle la dimension orchestrale de l'instrument. C'est un instrument qui a une richesse sonore qui permet de faire tellement de choses. Quelque part, je suis un violoniste ou un altiste frustré. J'ai toujours cherché à pouvoir tenir le son, à travailler dans la matière du son même ; l'attaque du son m'intéresse peu. C'est la vie du son qui m'intéresse. Donc, quelque part il manque l'archet, qui permet de faire des nuances, de tenir le son. D'où mon intérêt d'utiliser la pédale de volume pour pouvoir aider, et faire par exemple des échos pour superposer. Beaucoup de mes manières d'utiliser les effets, c'est pour essayer de s'approcher d'un archet et éventuellement de la pédale du piano. Parce que la guitare, dès que tu lâches la corde, ne donne plus rien. Donc si j'ai beaucoup de manières de me servir de la pédale de volume, c'est pour pouvoir avoir du son qui tienne plus longtemps, qui tienne mieux pour faire des nuances, c'est de cette façon qu'il vit ; sinon, la guitare électrique, c'est une corde pincée. Je pense que c'est pour cela que j'ai fait de la musique contemporaine, spécialement avec la génération de l'ensemble de l'Itinéraire. La vie du son, nos centres d'intérêt allaient dans ce sens.

En fait, je n'ai pas de technique particulière. C'est ouvert, il m'est très difficile de définir ma technique. J'ai des techniques, des manières de me servir de l'instrument. C'est vraiment axé sur la sensation. Je me bats avec mes élèves pour leur faire comprendre cela. Les gens qui vont plus tard t'engager, t'écouter, la manière dont tu t'y prends ils s'en fichent, ça sonne, c'est agréable, c'est sympa à écouter, ou ils s'emm... On peut parler de technique entre nous, mais devant le chef d'orchestre, s'il 
va te demander de jouer un peu plus lié, la façon dont tu le fais, il s'en fiche, c'est à toi de trouver les solutions pour le faire.

Quant au répertoire écrit, déjà créé, se posent des questions d'interprétation...

Claude Pavy: Il y a de grands types de sonorités, les sons clairs, les sons ronds, la distorsion, les distorsions très bzzi, d'autres plus chaudes... Finalement, il n'y en a pas tant que cela. Après, on applique des enrichissements, un peu de chorus, des choses comme ça. Il faut que les interprètes apprennent comment cela fonctionne, les principes de base. Ensuite, c'est à chacun, avec le matériel qu'il a, d'aller dans le sens de la sonorité qui est prévue. Aujourd'hui même, je n'ai pas le matériel d'il y a vingt ans, mais si je dois reprendre une pièce ancienne, la démarche est la même. Avec le matériel de maintenant, je vais retrouver les sonorités de l'époque, connaissant comment fonctionne un chorus, une distorsion. Il ne s'agit pas de retrouver le son d'origine, c'est impossible. Mais c'est pareil avec le concerto de Sibelius, si tu as quatre violonistes, tu as quatre sons différents. Si tu veux le même son, tu mets le disque, là c'est fixé. Si on fait de la musique vivante en direct, il y aura toujours des différences, et en plus il y a la personnalité de celui qui joue. Et c'est de la musique qui est jouée, il se passe quelque chose ou pas, c'est là l'essentiel.

Ce fameux problème du son, on le retrouve à tous les niveaux. Le guitariste qui dit : «je veux LE son », qu'est-ce que cela veut dire? Le son en soi, cela n'a pas de sens. Tout est son. De nombreux guitaristes électriques passent leur temps à courir après la disto qui tue. Cela n'a de sens que dans un style, un contexte musical, équilibré dans une orchestration. Le son, à l'écoute, va être pertinent dans un contexte, un milieu esthétique, énergique, lié au caractère de la pièce. Deux exemples: un orchestre symphonique avec un beau tapis de cordes à la Mahler. Et tout à coup tu as un hautbois aérien, fin, planant qui se mêle aux cordes. Le même hautbois tout seul, c'est une horreur, raide, agressif, pas agréable du tout. Autre exemple vécu. À l'occasion d'un concert de charité où j'accompagnais sur scène Yves Montant, j'ai croisé Santana lors des répétitions, nous avons échangé nos guitares, et j'ai donc joué sur la guitare de Santana, sur ses amplis, avec ses réglages. Je n'avais pas le son de Santana. Et lui, quand il a pris ma guitare, mes amplis et mes réglages, cela ressemblait encore à du Santana. Bien sûr il y a les boutons sur des appareils, mais c'est juste pour le traitement du son. La création du son, c'est toi, et ce qui se passe dans tes doigts.

\section{AUTEURS}

\section{SANTIAGO QUINTANS}

Coordinateur du programme Jazz au conservatoire (CRD) du Mans, compositeur. Doctorant au CICM université Paris 8

OTSO LÄHDEOJA

CICM, université Paris 8, MSH Paris Nord 